\title{
Enhanced vortex pinning by a composite antidot lattice in a superconducting $\mathbf{P b}$ film
}

\author{
A. V. Silhanek, ${ }^{1, *}$ L. Van Look, ${ }^{2}$ R. Jonckheere, ${ }^{2}$ B. Y. Zhu, ${ }^{1, \dagger}$ S. Raedts, ${ }^{1}$ and V. V. Moshchalkov ${ }^{1}$ \\ ${ }^{1}$ Nanoscale Superconductivity and Magnetism Group, Laboratory for Solid State Physics and Magnetism, K. U. Leuven, \\ Celestijnenlaan 200 D, B-3001 Leuven, Belgium \\ ${ }^{2}$ IMEC vzw, Kapeldreef 75, B-3001 Leuven, Belgium
}

(Received 14 October 2004; revised manuscript received 31 March 2005; published 5 July 2005)

\begin{abstract}
The use of artificial defects is known to enhance the superconducting critical parameters of thin films. In the case of conventional superconductors, regular arrays of submicron holes (antidots) substantially increase the critical temperature $T_{c}(H)$ and critical current $I_{c}(H)$ for all fields. Using electrical transport measurements, we study the effect of placing an additional small antidot in the unit cell of the array. This composite antidot lattice consists of two interpenetrating antidot square arrays with a different antidot size and the same lattice period. The smaller antidots are located at the centers of the cells of the large antidots array. We show that the composite antidot lattice can trap a higher number of flux quanta per unit cell inside the antidots compared to a reference antidot film without the additional small antidots. As a consequence, the field range in which an enhanced critical current is observed is considerably expanded. Finally, the possible stable vortex lattice patterns at several matching fields are determined by molecular-dynamics simulations.
\end{abstract}

DOI: 10.1103/PhysRevB.72.014507

PACS number(s): 74.78.Db, 74.25.Fy, 74.25.Qt

\section{INTRODUCTION}

During the past decade, compelling evidence has shown that the introduction of an array of microholes (antidots) in a superconducting film has a profound influence on both the critical current ${ }^{1,2} I_{c}(H)$ and the critical temperature ${ }^{3,4}$ $T_{c}(H)$. Typically, at temperatures used for transport measurements, the antidots are able to trap only one flux quantum $\Phi_{0}$ before saturation sets in. In this case, after the first matching field $H_{1}$, interstitial vortices appear in the sample, creating a "composite vortex lattice," where part of the vortices is strongly pinned at the antidots and the rest occupies interstitial positions in between the antidots. ${ }^{5}$ Due to their higher mobility, the presence of interstitial vortices lowers the critical current $I_{c}(H)$ significantly and broadens the $R(T)$ transition.

In this work, we study a composite antidot array, consisting of two interpenetrating square lattices with the same period $d=1.5 \mu \mathrm{m}$ but different antidot size $\left(a_{1}\right.$ $=0.55 \mu \mathrm{m}$ and $\left.a_{2}=0.25 \mu \mathrm{m}\right)$. The two sublattices are shifted with respect to each other by half a unit cell along $x$ and $y$ directions, so that the small antidot is placed in the center of the unit cell of the lattice of large antidots (see Fig. 1). This arrangement of antidots corresponds to the vortex lattice configuration at the second matching field in a sample with a single square array of antidots with $n_{s}=1$. The purpose of this experiment is to enlarge the field range where an enhanced critical current $I_{c}(H)$ is observed by having efficient pinning sites exactly at the locations where the interstitial vortices would appear if the smaller antidots were not present.

\section{EXPERIMENTAL DETAILS}

The used sample is a 50-nm-thick $\mathrm{Pb}$ film with a composite antidot lattice. The results obtained with this sample are directly contrasted with those measured on a reference antidot sample without the small holes, i.e., $a_{1}=0.5 \mu \mathrm{m}$ and $a_{2}=0 \mu \mathrm{m}$. In both cases, the bridge made for transport measurements [see Fig. 1(a)] has a width of $300 \mu \mathrm{m}$ and a voltage contact separation of $2 \mathrm{~mm}$. The unit cell of the composite antidot array is shown schematically in Fig. 1(b). The procedures followed to grow the samples are described in Ref. 6. The transport measurements were carried out in a commercial PPMS-Quantum Design device with a temperature stability better than $0.5 \mathrm{mK}$. All measurements were
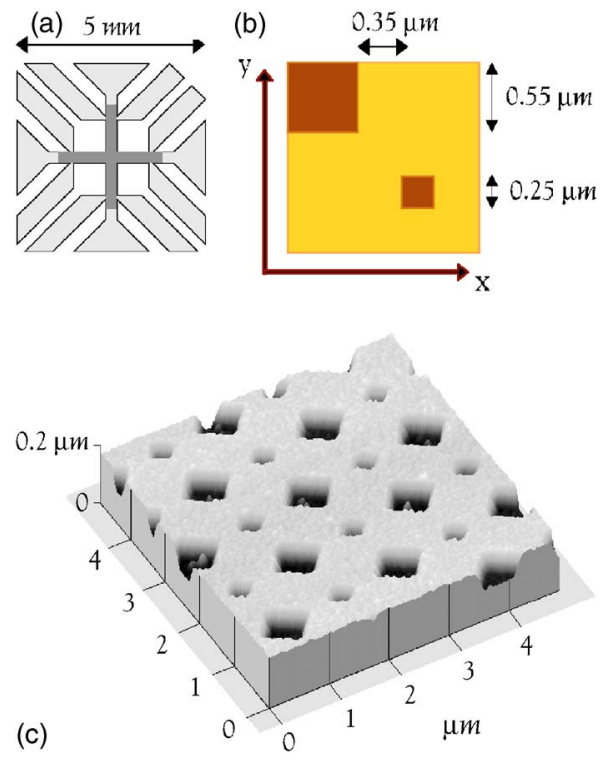

FIG. 1. (Color online) Layout of the $\mathrm{Pb}$ film with a composite array of square antidots of two different sizes. (a) Geometry of the sample showing the patterned area in dark gray. (b) Schematic presentation of a unit cell of the antidot array. (c) Atomic force micrograph of a $5 \times 5 \mu \mathrm{m}^{2}$ area of the composite antidot array. The lattice period $d$ is $1.5 \mu \mathrm{m}$; the antidot sizes are $a_{1}=0.55 \mu \mathrm{m}$ and $a_{2}=0.25 \mu \mathrm{m}$. 
performed with the field $\mathbf{H}$ applied perpendicular to the surface of the film.

From a resistivity of $\rho(7.5 \mathrm{~K})=5.33 \mu \Omega \mathrm{cm}$, we estimate an elastic mean free path of $\ell=9 \mathrm{~nm}$, and therefore a superconducting coherence length $\xi(0)=25 \mathrm{~nm}$ in the dirty limit. These values are smaller than those obtained for the reference antidot sample $[\ell=27 \mathrm{~nm}$ and $\xi(0) \approx 40 \mathrm{~nm}]$. Since in a film without antidots coevaporated with the sample containing the composite antidot we obtained $\ell=27 \mathrm{~nm}$, this difference seems to be caused by the more complex lift-off procedure due to the presence of the small holes.

Knowing the mean free path $\ell$ and using the London penetration depth for the bulk $\mathrm{Pb}$, we obtain $\lambda(0)=71 \mathrm{~nm}$. Due to the perforation, the effective penetration depth increases, and therefore $\lambda$ should be modified according to ${ }^{8}$ $\Lambda(0)=\lambda(0) / \sqrt{1-2\left(S_{a} / S_{t}\right)}=86 \mathrm{~nm}$, where $S_{a}$ and $S_{t}$ are the area of the holes and the total area per unit cell, respectively. As a result, the Ginzburg-Landau parameter $\kappa$ amounts to $\kappa=\Lambda(0) / \xi(0)=3.4>1 / \sqrt{2}$, and therefore this sample is a type-II superconductor. ${ }^{9}$

The sample has been characterized by means of atomic force microscopy. An atomic force microscopy topograph of a $5 \times 5 \mu \mathrm{m}^{2}$ area of the film containing a composite antidot lattice is shown in Fig. 1(c). The rootmean-square roughness on a $1 \mu \mathrm{m}^{2}$ area of the sample in between the antidots is $\sigma_{\mathrm{rms}}=3 \mathrm{~nm}$. This value is about two times larger than for the plane film and the reference sample with antidots. This difference reinforces the idea that the film with the composite antidot lattice has suffered a small degradation due to a more complicated lift-off procedure.

\section{RESULTS AND DISCUSSION}

\section{A. Superconducting phase boundary}

We have measured the critical temperature $T_{c}(H)$ as a function of field for the sample with a composite antidot lattice. The results obtained with a resistance criterion of $10 \%$ of the normal state resistance $R_{n}$ and a measuring current of $I_{\mathrm{ac}}=10 \mu \mathrm{A}$ are shown in Fig. 2, together with the phase boundary obtained for the reference antidot film. The solid line depicts the expected upper critical field boundary of a plane film with the same coherence length as the reference antidot sample according to $H_{c 2}=\Phi_{0} / 2 \pi \xi(T)^{2}$. The measured boundary of the reference antidot film is close to the $H_{c 3}(T)$ line corresponding to the surface nucleation of superconductivity around the holes, whereas the solid line represents the bulk superconducting transition $H_{c 2}(T)$.

Due to the presence of the antidot array, matching features appear in $T_{c}(H)$ with a periodicity of $H_{1}=\Phi_{0} / d^{2}=9.2 \mathrm{G}$, corresponding to the lattice parameter $d=1.5 \mu \mathrm{m}$. Local maxima are visible in the $T_{c}(H)$ of the composite array for all integer matching fields $H_{n}(n=1,2, \ldots, 6)$, whereas no evidence of rational matching features is observed. Thus, the addition of the extra antidot in the center of the unit cell of the array with large antidots leaves the matching period

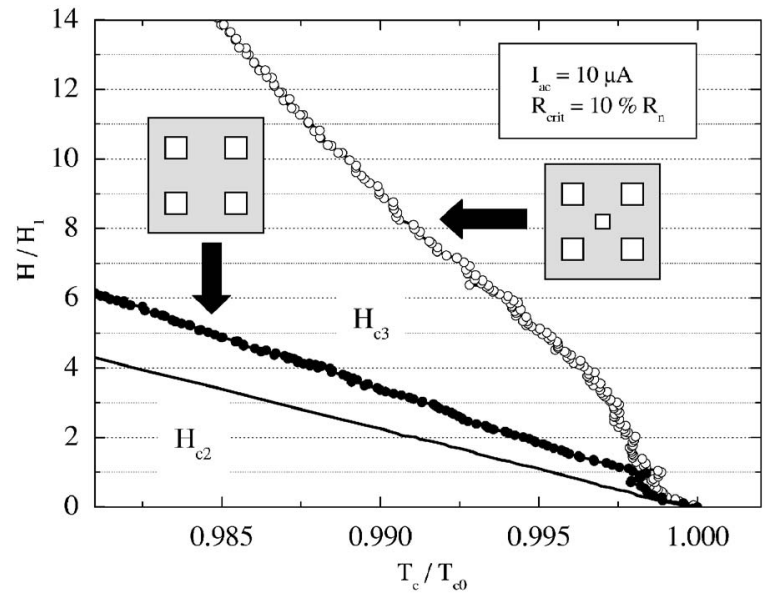

FIG. 2. $T_{c}(H)$ phase boundary for the film patterned with a composite antidot array, measured with an ac current of $I_{\mathrm{ac}}=10 \mu \mathrm{A}$ and a resistance criterion of $R_{\text {crit }}=10 \% R_{n}$ (filled symbols). The open symbols show the phase boundary obtained for the reference antidot sample using the same criterion. The solid line is the calculated linear $T_{c}(H)$ phase boundary for a plane film with the same coherence length $\xi(0)=40 \mathrm{~nm}$ as the antidot patterned film. The field axis is normalized to the first matching field $H_{1}=9.2 \mathrm{G}$. The temperature axis is normalized to $T_{c 0}$, the transition temperature at $H=0$.

unchanged. This is an important observation, since the composite antidot lattice can also be regarded as a square lattice, tilted by $45^{\circ}$, with a unit cell twice as small as that of the original lattice. If this were the periodicity felt by the vortices, the matching period would amount to $18.4 \mathrm{G}$, which is twice as large as the observed period. In that case, one would expect the local maxima at even matching fields $H_{n}(n=2,4, \ldots)$ in Fig. 2 to be more pronounced than the ones at odd matching fields $H_{n}(n=1,3, \ldots)$. Since this is not the case, we conclude that all these peaks correspond to integer matching fields, indicating that the main period felt by the vortices is the period of the lattice with large antidots.

Further information can be gained from the $R(T)$ transition width $\Delta T_{c}(H)=T_{c}\left(R_{\text {crit }}=97 \% R_{n}\right)-T_{c}\left(R_{\text {crit }}\right.$ $=0.1 \% R_{n}$ ) as a function of $H$, as shown in Fig. 3 (filled symbols). In this plot, three different regimes can be clearly distinguished. For $H<H_{4}$, the coherence length is larger than the width of the strands, thus leading to a parabolic background in the $T_{c}(H)$ phase boundary. In this so-called "collective" regime, we observe that the $R(T)$ transition width remains almost constant. For fields higher than $H_{4}$, an increase of the transition width can be observed, superposed with matching features at $H_{5}$ and $H_{6}$. We interpret the sudden increase in the transition width as a crossover to the regime where interstitial vortices appear in the sample.

The interstitial regime is indicated by the gray area in Fig. 3 for the composite array. This regime ranges up to $3.6 \xi(T)=d-a$, i.e., up to $\sim H_{8}$, where a change in the $\Delta T_{c}(H)$ slope can be observed. For higher fields, the single object regime is entered, where a linear phase boundary slightly distorted by an oscillation with period ${ }^{4} H^{*}=\Phi_{0} / a_{1}^{2}$ 


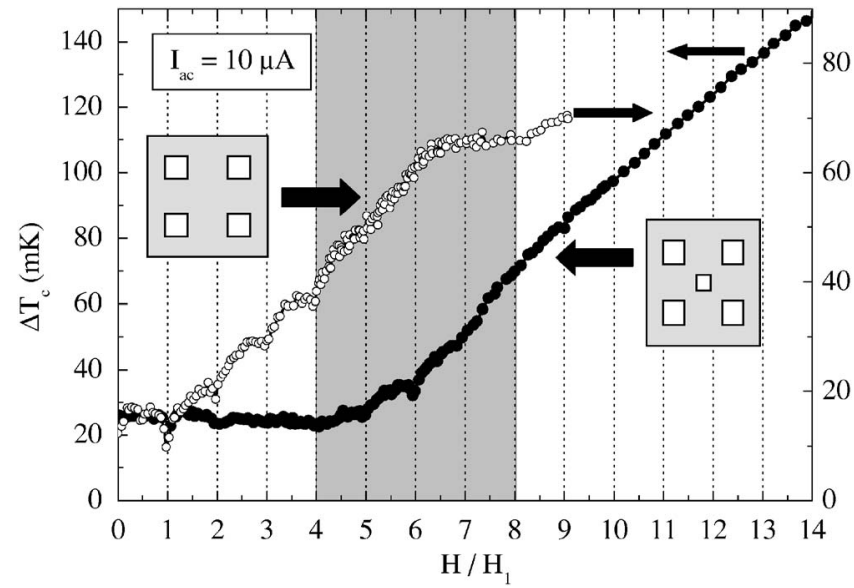

FIG. 3. Filled symbols: transition width $\Delta T_{c}(H)=T_{c}\left(R_{\text {crit }}\right.$ $\left.=97 \% R_{n}\right)-T_{c}\left(R_{\text {crit }}=0.1 \% R_{n}\right)$ of the film with a composite antidot array, measured with a current of $I_{\mathrm{ac}}=10 \mu \mathrm{A}$. The gray box marks the "interstitial" regime for the composite array. Open symbols: transition width $\Delta T_{c}(H)=T_{c}\left(R_{\text {crit }}=99 \% R_{n}\right)-T_{c}\left(R_{\text {crit }}\right.$ $\left.=0.1 \% R_{n}\right) \quad$ of the reference antidot film for $I_{\mathrm{ac}}=10 \mu \mathrm{A}$. The thin black arrows indicate the ordinate scale for each curve.

$\sim 69 \mathrm{G}$ is expected. Although the linear phase boundary is indeed observed, single object oscillations are difficult to resolve in the narrow field range investigated. For comparison, in the same figure we show $\Delta T_{c}(H)=T_{c}\left(R_{\text {crit }}\right.$ $\left.=99 \% R_{n}\right)-T_{c}\left(R_{\text {crit }}=0.1 \% R_{n}\right)$ for the reference antidot sample (open symbols). From this curve, it is easy to see that if the smaller additional antidots are absent, the crossover to the interstitial regime occurs at $H \sim 1.5 H_{1}$. Therefore, the presence of the additional smaller antidots has substantially delayed the appearance of interstitial vortices. From the $\Delta T_{c}(H)$ curve, we thus conclude that the total number of trapped flux quanta per unit cell of the antidot lattice is at least four.

The following scenario explains the observed behavior. Up to $H_{1}$, the vortices will be attracted towards the large antidots. Between $H_{1}$ and $H_{2}$, vortices begin to occupy the small antidots. Due to their size, these small antidots trap at most a single quantum vortex. Therefore, they will be completely saturated at $\mathrm{H}_{2}$, creating a repulsive potential at the position of the small antidot. Figure 4 shows a schematic evolution of the potential landscape along a diagonal of the array (see the dashed line in the inset) that would be experienced by a vortex for $H=0, H_{1}$, and $H_{2}$. Since the large antidots pin one flux quantum, at $H=H_{1}$ a surface barrier has emerged at the antidot edges. For $H=H_{2}$, the contribution to the potential of the small antidot at the center of the unit cell is strongly repulsive. When additional vortices enter the sample, they will be pushed towards the large antidots, leading to an increase of their effective saturation number. In other words, the additional repulsive potential at the small antidots helps to increase the saturation number of the larger antidots. We therefore conclude that of the four flux quanta trapped per unit cell of the composite antidot lattice, one is pinned by the small antidot while three are pushed into the larger holes. This leads to a substantial broadening of the

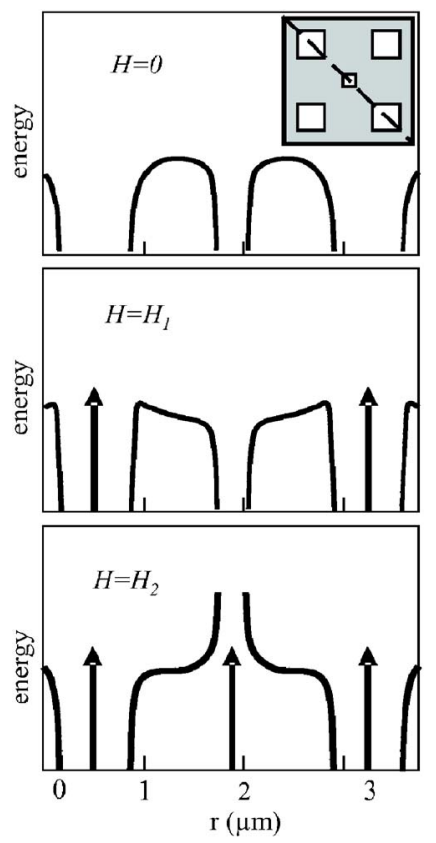

FIG. 4. Schematic representation of the potential along a diagonal of the composite antidot array (see inset), experienced by a vortex entering the sample for $H=0, H_{1}$, and $H_{2}$.

field range where a strong $T_{c}(H)$ enhancement is observed. A similar picture was introduced by Doria and co-workers to explain the multiple trapping of vortices at high fields, as a result of the pressure exerted by the external vortices into the pinning site. ${ }^{10}$

\section{B. Critical current}

So far we have explored the normal-superconducting boundary. Let us now turn to isothermal critical current measurements in order to study the vortex dynamics within the superconducting state. The critical current as a function of field $I_{c}(H)$ was measured using a $100 \mu \mathrm{V}$ voltage criterion for several temperatures close to $T_{c}(H)$. The results, in order of decreasing temperature, are shown in Figs. 5 and 6. The absolute value of the critical current density at zero field for the composite antidot array amounts to $I_{c o}=I_{c}(H=0)$ $=6.8 \times 10^{8}\left(\mathrm{~A} / \mathrm{m}^{2}\right)$ at $T / T_{c 0}=0.974$. This value is a factor $\sim 3$ lower than the critical current density obtained for the reference antidot lattice, in part due to the specific geometry of the lateral nanopatterning which influences the current distribution throughout the film, hereby also affecting the critical current $I_{c o}$. In order to compare the pinning properties of the film with the composite antidot lattice (open symbols) and the reference antidot lattice (solid lines) measured at the same reduced temperature, we have normalized the critical current by $I_{c o}$. Notice that since the saturation number $n_{s}$ is mainly determined by the coherence length ${ }^{11,12} \xi(T)$, which in turn depends solely on the reduced temperature $t=T / T_{c}$, it is enough to compare the results obtained on these samples at the same reduced temperature, without the necessity of normalizing the field.

The $I_{c}(H) / I_{c 0}$ curves for the film with a composite antidot array (Figs. 5 and 6) have been measured for negative fields 

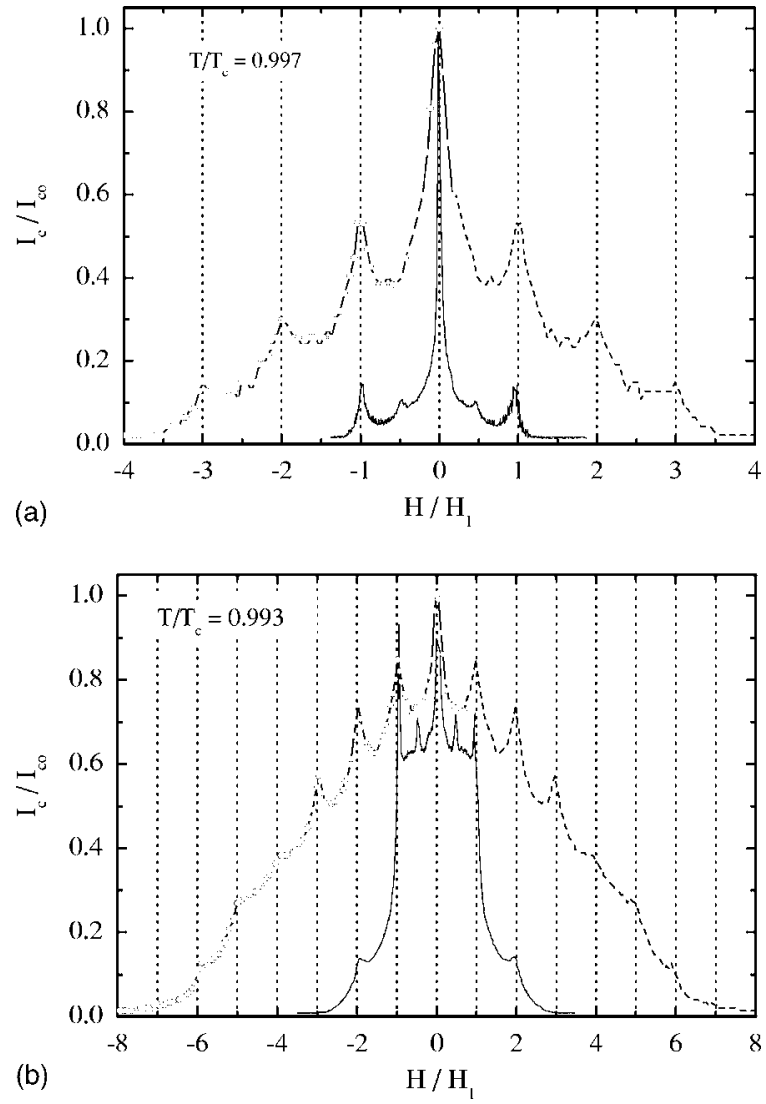

FIG. 5. Normalized critical current at $T / T_{c 0}=0.997$ and $T / T_{c 0}=0.993$ of a film with a composite antidot array. The curves were measured for $H<0$ (open symbols) and symmetrized for clarity for $H>0$ (dashed line). For comparison, the solid line shows the normalized critical current $I_{c}(H) / I_{c 0}$ obtained for the film with the reference antidot lattice.

(open symbols) and symmetrized for $H>0$ for clarity (dashed lines). All curves show distinct periodic matching features, with a period $H_{1}$ corresponding to the unit cell of the lattice with the large (or the small) antidots $(d=1.5 \mu \mathrm{m})$. In the upper panel of Fig. $5\left(T / T_{c 0}=0.997\right)$, the $I_{c}(H) / I_{c 0}$ curve for the film with a composite antidot lattice shows sharp maxima at $H_{1}, H_{2}$, and $H_{3}$. This behavior is expected at temperatures sufficiently close to $T_{c 0}$, where it is not possible to have interstitial vortices in the superconducting strands between the antidots. As we mentioned above, interstitial vortices appear in the sample only for $T / T_{c 0} \leqslant 0.994$. Accordingly, at a lower temperature, $T / T_{c 0}=0.993$ (Fig. 5, lower panel), and all temperatures below that (Fig. 6), a strong enhancement of $I_{c}(H) / I_{c 0}$ in the film with a composite antidot lattice can be found for fields higher than the first matching field $H_{1}$, compared to the reference antidot lattice. The reason for this lies in the ability of the composite antidot lattice to pin more flux quanta inside the antidots compared to the reference antidot array. It should be noted that the field range where the film has a finite critical current, i.e., where the film remains superconducting, is considerably broader for the composite than for the reference antidot array.

The appearance and sharpness of the matching features in the $I_{c}(H) / I_{c 0}$ curves are temperature-dependent. At
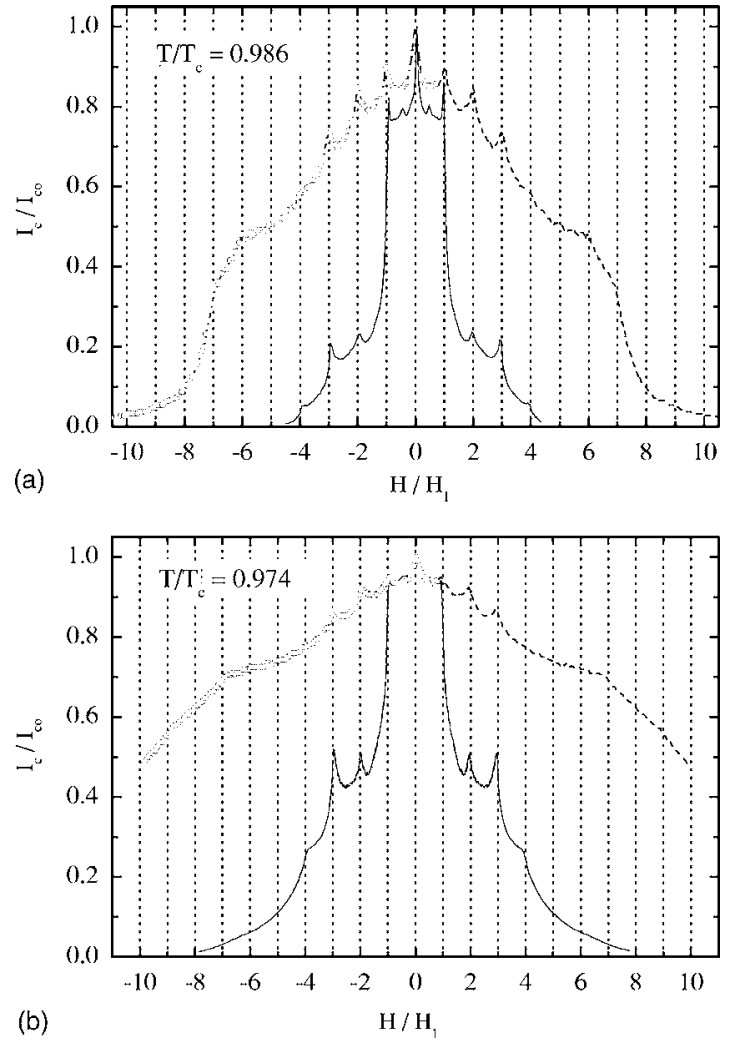

FIG. 6. Normalized critical current at $T / T_{c 0}=0.986$ and $T / T_{c 0}=0.974$ of a film with a composite antidot array. The curves were measured for $H<0$ (open symbols) and symmetrized for clarity for $H>0$ (dashed line). For comparison, the solid line shows the normalized critical current $I_{c}(H) / I_{c 0}$ obtained for the film with the reference antidot lattice.

$T / T_{c 0}=0.993$ (Fig. 5, lower panel), every integer-matching peak up to $H_{6}$ can be clearly seen. The maxima at $H_{1}, H_{2}$, and $H_{3}$ are very pronounced. At $H_{4}$ and $H_{5}$, one finds cusps rather than local maxima in $I_{c}(H) / I_{c 0}$. The matching feature at $H_{6}$ is again peaklike. This indicates that the vortex patterns formed at $H_{4}$ and $H_{5}$ are less stable than the vortex configuration at $H_{6}$.

When the temperature is lowered down to $T / T_{c 0}=0.986$ (Fig. 6, upper panel), we find again sharp matching features in $I_{c}(H) / I_{c 0}$ at $H_{1}, H_{2}$, and $H_{3}$, and only very weak cusps at $H_{4}$ and $H_{5}$. At $H_{6}$, the local maximum has developed into a pronounced cusp, after which a substantial change in the $I_{c}(H) / I_{c 0}$ slope occurs. A second smaller slope change can be found at $H_{7}$. At the lowest measured temperature, $T / T_{c 0}=0.974$ (Fig. 6, lower panel), the only matching features left are the sharp local maxima at $H_{1}, H_{2}$, and $H_{3}$, and one pronounced cusp at $H_{7}$. It appears that, at this temperature, the seventh matching field $H_{7}$ plays the same role as the sixth matching field $H_{6}$ at $T / T_{c 0}=0.986$. This fact indicates that at $T / T_{c 0}=0.974$, the total number of trapped flux quanta per unit cell of the composite lattice increases from four to five.

It is worth noticing that the normalized critical current at the first matching field $H_{1}$ reaches approximately the same value for the film with the composite and with the reference antidot lattice, except for the $I_{c}(H) / I_{c 0}$ curve taken at 

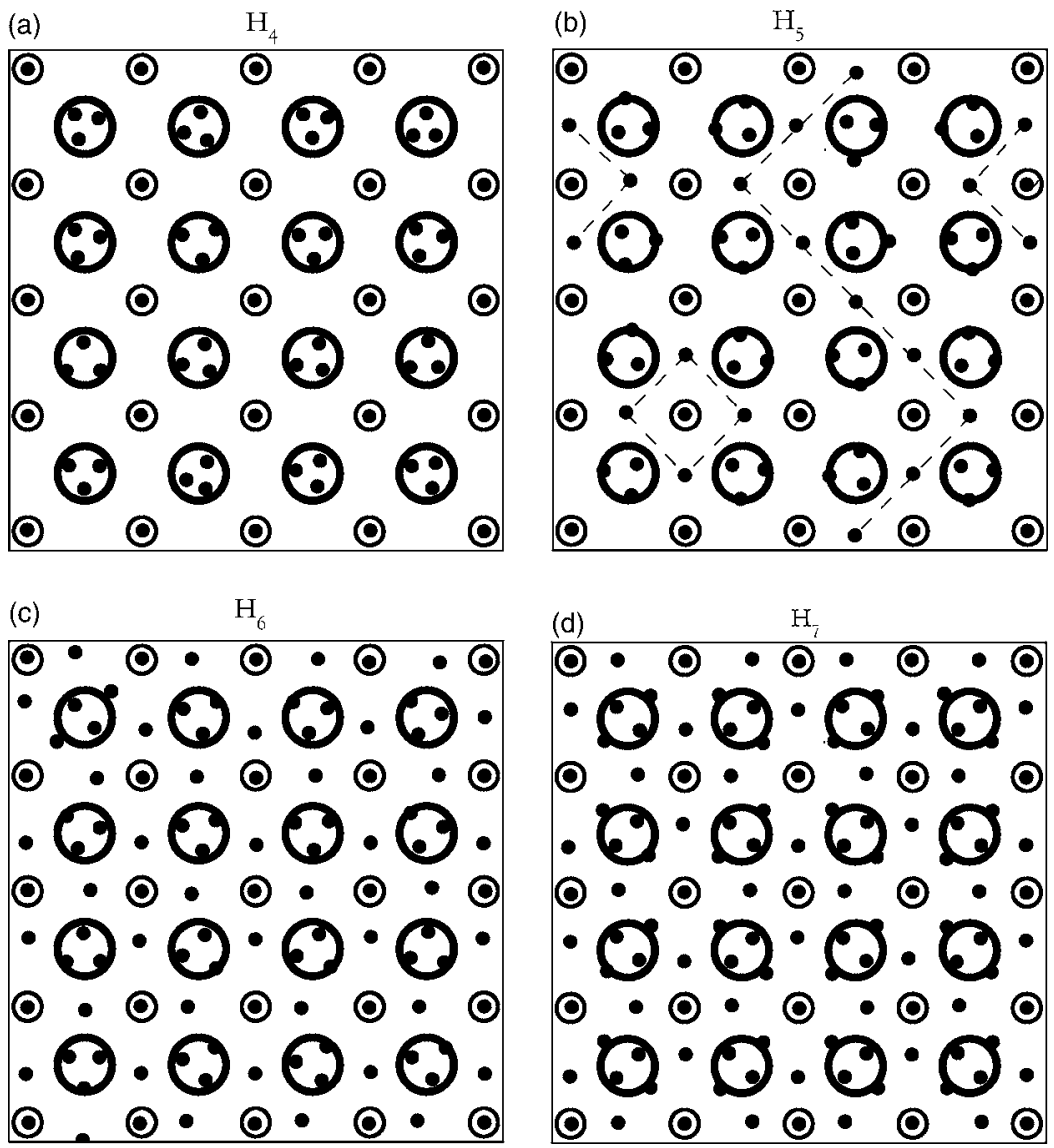

FIG. 7. Suggested vortex pattern at $H_{4}, H_{5}, H_{6}$, and $H_{7}$. All patterns have been obtained by molecular-dynamics simulations by an annealing procedure, except the one at $H_{4}$. Open circles and black dots represent pinning sites and single quantum vortices, respectively.

$T / T_{c 0}=0.997$. This fact makes the film with the reference antidot array a good candidate to compare its pinning properties with those of the composite antidot array.

\section{Stable vortex patterns}

We have performed molecular-dynamics simulations to obtain the vortex patterns at the matching fields $H_{5}, H_{6}$, and $H_{7}$. To model the composite vortex lattice, two interpenetrating arrays of Gaussian sites with a different radius and a different pinning force were used. Figure 7 shows the vortex configurations we suggest for $H_{4}, H_{5}, H_{6}$, and $H_{7}$. Multiquanta vortices are represented in this model by a multiple occupation of a pinning site with (repulsive) single-quantum vortices. Since in the experiment the pinning sites consist of real holes in the film, the vortices trapped in the same pinning site will be interpreted as multiquanta vortices, even though they are depicted as separate single flux quantum entities in the plots.

The vortex pattern at $H_{4}$, which is drawn schematically and was not calculated, depicts all antidots occupied with the maximum number of vortices. The large antidots trap $3 \Phi_{0}$ vortices; the smaller antidots trap a $\Phi_{0}$ vortex. No interstitial vortices are present in the sample. At $H_{5}$, there is one interstitial vortex present per unit cell of the array. There can be seen a tendency of the interstitial vortices to form diagonal lines, which make zigzag traces across the sample, indicated by dashed lines in Fig. 7(b). Thus the obtained pattern is consequently not very stable.

At $H_{6}$, a highly symmetric vortex pattern is formed. In this case, two interstitial vortices are present per unit cell, which are positioned approximately at the center of the line connecting two neighboring large antidots. Due to its high symmetry, the vortex pattern at $H_{6}$ is very stable. This result is consistent with the fact that the matching features at $H_{6}$ in $T_{c}(H)$ or $I_{c}(H) / I_{c 0}$ are more pronounced than at $H_{5}$ (see, e.g., Figs. 3 and 6, upper panel). At $H_{7}$, the calculations were not able to produce a regular vortex pattern with an occupation of three at the large pinning sites and one at the smaller pinning sites. This indicates that the stability of a vortex pattern at $H_{7}$ is not very high. ${ }^{13,14}$

\section{CONCLUSIONS}

We have used a composite antidot lattice, consisting of two interpenetrating antidot arrays with a different antidot size, but with the same lattice period, as a strong periodic pinning potential for the vortex lattice in a superconducting film. The shift between the two lattices is such that the smaller antidots are situated exactly at the centers of the cells of the array of large antidots. We have shown that this pin- 
ning array can stabilize the vortex lattice at several matching fields from $H_{1}$ to $H_{7}$.

Measurements of the critical temperature $T_{c}(H)$ and current $I_{c}(H) / I_{c 0}$ as a function of magnetic field have demonstrated that the composite antidot lattice can trap a considerably higher amount of flux quanta per unit cell inside the antidots, compared to a reference antidot film without the additional small antidots. This means that the appearance of interstitial vortices in the composite antidot lattice is delayed to higher magnetic fields. The presence of the smaller antidots has therefore increased the effective saturation number of the large antidots, which has led to a considerable expansion of the field range in which an enhanced critical current is observed.

\section{ACKNOWLEDGMENTS}

This work was supported by the Research Fund K.U. Leuven GOA/2004/02, the Belgian Interuniversity Attraction Poles (IUAP), the Fund for Scientific Research Flanders (FWO), and the ESF "VORTEX" program.
*Present address: MST-NHMFL, MS E536, Los Alamos National Laboratory, Los Alamos, NM 87544, USA.

†Present address: National Laboratory for Superconductivity, Institute of Physics, Chinese Academy of Sciences, Beijing 100080, China.

${ }^{1}$ V. V. Moshchalkov, M. Baert, E. Rosseel, V. V. Metlushko, M. J. Van Bael, and Y. Bruynseraede, Physica C 282, 379 (1997); V. V. Moshchalkov, M. Baert, V. V. Metlushko, E. Rosseel, M. J. Van Bael, K. Temst, Y. Bruynseraede, and R. Jonckheere, Phys. Rev. B 57, 3615 (1998).

${ }^{2}$ V. Metlushko, U. Welp, G. W. Crabtree, R. Osgood, S. D. Bader, L. E. DeLong, Zhao Zhang, S. R. J. Brueck, B. Ilic, K. Chung, and P. J. Hesketh, Phys. Rev. B 60, R12 585 (1999).

${ }^{3}$ U. Welp, Z. L. Xiao, J. S. Jiang, V. K. Vlasko-Vlasov, S. D. Bader, G. W. Crabtree, J. Liang, H. Chik, and J. M. Xu, Phys. Rev. B 66, 212507 (2002).

${ }^{4}$ E. Rosseel, T. Puig, M. Baert, M. J. Van Bael, V. V. Moshchalkov, and Y. Bruynseraede, Physica C 282, 1567 (1997).

${ }^{5}$ C. Reichardt and N. Gronbech-Jensen, Phys. Rev. Lett. 85, 2372 (2000).

${ }^{6}$ S. Raedts, A. V. Silhanek, M. J. Van Bael, and V. V. Mosh- chalkov, Physica C 404, 298 (2004).

${ }^{7}$ T. P. Orlando and K. A. Delin, Foundations of Applied Superconductivity (Addison-Wesley, Reading, MA,1991).

${ }^{8}$ A. Wahl, V. Hardy, J. Provost, Ch. Simon, and A. Buzdin, Physica C 250, 163 (1995).

${ }^{9}$ In addition to the perforation effect, the finite thickness $\delta$ of the film also yields an increase of the effective penetration depth up to $\Lambda=2 \lambda^{2} / \delta$, therefore giving rise to an even higher $\kappa$ value. This issue has been briefly addressed in V. V. Moshchalkov, M. Baert, V. V. Metlushko, E. Rosseel, M. J. Van Bael, K. Temst, R. Jonckheere, and Y. Bruynseraede, Phys. Rev. B 54, 7385 (1996).

${ }^{10}$ M. M. Doria and G. F. Zebende, Phys. Rev. B 66, 064519 (2002).

${ }^{11}$ G. S. Mkrtchyan and V. V. Schmidt, Sov. Phys. JETP 34, 195 (1972).

${ }^{12}$ H. Nordborg and V. M. Vinokur, Phys. Rev. B 62, 12408 (2000).

${ }^{13}$ B. Y. Zhu, L. Van Look, V. V. Moshchalkov, B. R. Zhao, and Z. X. Zhao, Phys. Rev. B 64, 012504 (2001).

${ }^{14}$ S. Raedts, A. V. Silhanek, M. J. Van Bael, and V. V. Moshchalkov, Phys. Rev. B 70, 024509 (2004). 